\title{
ON THE CHANG AND COOPER SCHEME APPLIED TO A LINEAR FOKKER-PLANCK EQUATION*
}

\author{
CHRISTOPHE BUET ${ }^{\dagger}$ AND STÉPHANE DELLACHERIE ${ }^{\ddagger}$
}

\begin{abstract}
We show that for a particular linear Fokker-Planck operator, the explicit Chang and Cooper scheme is positive and entropy satisfying under a CFL criterion when the initial condition is positive. Then, we deduce that the distribution given by the explicit Chang and Cooper scheme converges toward a discrete Maxwellian equilibrium.
\end{abstract}

Key words. Kinetic equation, Fokker-Planck-Landau operator, explicit Chang and Cooper scheme, positive and entropy satisfying scheme.

AMS subject classifications. 65M06, 65M12, 82C40, 82D10.

\section{Introduction}

The Chang and Cooper scheme [1] is a classical scheme $[2,3,4,5]$ used to solve a spatially homogeneous kinetic equation of the type

$$
\frac{\partial f}{\partial t}=S(f)
$$

where $f(t, v)\left((t, v) \in \mathbb{R}_{+} \times \mathbb{R}^{3}\right)$ is a non-negative distribution and where $S(f)$ is a linear or non-linear Fokker-Planck operator. The main property of the Chang and Cooper scheme is that the numerical fluxes used to discretize $S(f)$ are equal to zero when the distribution $f$ is equal to the equilibrium distribution $f_{e}$ of equation (1.1) (thus, $f_{e}$ is such that $\left.S\left(f_{e}\right)=0\right)$. In other words, this scheme preserves the equilibrium $f_{e}$ when it is reached. Nevertheless, up to now there does not exist any convergence proof for the Chang and Cooper scheme, even in the linear case, showing that $\lim _{t \rightarrow+\infty} f(t, v)=f_{e}(t, v)$ at the discrete level.

In this paper, we show that by using the explicit Chang and Cooper scheme to discretize the particular linear Fokker-Planck operator

$$
S(f)=\Omega \nabla_{v} \cdot\left[\left(v-U_{e}\right) f+\frac{T_{e}}{m} \nabla_{v} f\right],
$$

under a Courant-Friedrichs-Levy (CFL) criterion, the distribution $f$ converges in large time toward the Maxwellian equilibrium

$$
\mathcal{M}_{N, U_{e}, T_{e}}(v)=\frac{N}{\left(2 \pi T_{e} / m\right)^{3 / 2}} \exp \left[-\frac{m\left(v-U_{e}\right)^{2}}{2 T_{e}}\right]
$$

at the discrete level, the quantity $N \in \mathbb{R}_{+}^{*}$ being the macroscopic density given by $\int_{\mathbb{R}^{3}} f(v) d v$ (when $S(f)$ is given by (1.2), the equilibrium distribution $f_{e}$ is equal to $\left.\mathcal{M}_{N, U_{e}, T_{e}}\right)$. The physical quantities $U_{e} \in \mathbb{R}^{3}, T_{e} \in \mathbb{R}_{+}^{*}, m \in \mathbb{R}_{+}^{*}$, and $\Omega \in \mathbb{R}_{+}^{*}$ in (1.2) are

\footnotetext{
${ }^{*}$ Received: December 2, 2009; accepted (in revised version): February 1, 2010. Communicated by François Golse.

${ }^{\dagger}$ CEA, DAM-DSSI-SNEC-LPNP, F-91680, Bruyères-le-Châtel, France (christophe.buet@cea.fr).

${ }^{\ddagger}$ CEA, Centre de Saclay, DEN-DM2S-SFME-LETR, F-91191 Gif-sur-Yvette, France (stephane. dellacherie@cea.fr).
} 
respectively the velocity of the medium, the temperature of the medium, the atomic mass of the particle, and the collision frequency of the particle on the medium. In the linear case, all these quantities are supposed to be constant. Let us underline that when $|v| \rightarrow+\infty$, we suppose that $\left(v-U_{e}\right) f(v)+\frac{T_{e}}{m} \nabla_{v} f \rightarrow 0$ because of the exponential decreasing of $f$. Then, by applying an integration by parts in $\int_{\mathbb{R}^{3}} S(f) d v$, we obtain that $N$ is also constant when $f$ is solution of (1.1). Let us note that the linear Fokker-Planck operator (1.2) is the linear version of the non-linear ion-electron collision operator studied in $[7,8]$ in which $U_{e}, T_{e}$, and $\Omega$ depend on the time. The explicit Chang and Cooper scheme based on the Chang and Cooper average (see Definition 2.1 below) applied to (1.2) is built in order to have $\left(v-U_{e}\right) f(v)+\frac{T_{e}}{m} \nabla_{v} f=0$ when $f=\mathcal{M}_{N, U_{e}, T_{e}}$ at the discrete level. To obtain the convergence result, we use the fact that, at the continuous level, $S(f)$ defined by the convection-diffusion operator (1.2) is equivalent to

$$
S(f)=\Omega \frac{T_{e}}{m} \nabla_{v} \cdot\left[\mathcal{M}_{N, U_{e}, T_{e}} \nabla_{v}\left(\frac{f}{\mathcal{M}_{N, U_{e}, T_{e}}}\right)\right] .
$$

The operator (1.4) is the non-logarithmic Landau formulation of (1.2). The key point of the convergence proof is to show that the Chang and Cooper scheme makes equivalent (1.2) and (1.4) at the discrete level. Let us note that $S(f)$ given by (1.2) is also equivalent to

$$
S(f)=\Omega \frac{T_{e}}{m} \nabla_{v} \cdot\left[f \nabla_{v} \log \left(\frac{f}{\mathcal{M}_{N, U_{e}, T_{e}}}\right)\right] .
$$

The operator (1.5) is the Landau formulation of $(1.2)$. In [7, 8], it was proven in the non-linear case that the scheme based on the entropic average (see Definition 2.3 below) makes equivalent (1.2) and (1.5) at the discrete level. A similar approach was proposed in [6] in the case of the non-linear isotropic ion-ion collision operator.

For the sake of simplicity, we define the Fokker-Planck operator $S(f)$ in monodimensional Cartesian geometry. Thus, the microscopic velocity $v$ belongs to $\mathbb{R}$, and we replace $\left(2 \pi T_{e} / m\right)^{3 / 2}$ with $\sqrt{2 \pi T_{e} / m}$ and $\nabla_{v}$ with $\partial_{v}$ in (1.2), (1.3), and (1.4), which means that (1.2) is now given by

$$
S(f)=\Omega \partial_{v}\left[\left(v-U_{e}\right) f+\frac{T_{e}}{m} \partial_{v} f\right]
$$

and that (1.3) and (1.4) are now given by

$$
\mathcal{M}_{N, U_{e}, T_{e}}(v)=\frac{N}{\sqrt{2 \pi T_{e} / m}} \exp \left[-\frac{m\left(v-U_{e}\right)^{2}}{2 T_{e}}\right]
$$

and

$$
S(f)=\Omega \frac{T_{e}}{m} \partial_{v}\left[\mathcal{M}_{N, U_{e}, T_{e}} \partial_{v}\left(\frac{f}{\mathcal{M}_{N, U_{e}, T_{e}}}\right)\right] .
$$

The velocity domain is discretized with $\left\{v_{j}\right\}_{j}$ where $j \in\left\{1, \ldots, j_{\max }\right\}\left(j_{\max }<+\infty\right)$. The velocity step is constant and equal to $\Delta v$. More precisely, we suppose that this domain is bounded and is equal to $\left[v_{1 / 2}, v_{j_{\max }+1 / 2}\right]$ with $\left(v_{1 / 2}, v_{j_{\max }+1 / 2}\right) \in \mathbb{R}_{-}^{*} \times \mathbb{R}_{+}^{*}$, and we define $v_{j}$ and $v_{j+1 / 2}$ with $v_{j}:=v_{1 / 2}+(j-1 / 2) \Delta v$ and $v_{j+1 / 2}:=v_{1 / 2}+j \Delta v$ 
where $\Delta v:=\left(v_{j_{\max }+1 / 2}-v_{1 / 2}\right) / j_{\max }$. Let us note that since $v_{1 / 2}>-\infty$ and $v_{j_{\max }+1 / 2}<$ $+\infty$, we will have to define particular boundary conditions in order to conserve the mass. The time subscript is $n$ and the time step is defined by $\Delta t$. Thus, we have $t^{n+1}=t^{n}+\Delta t$ knowing that $t^{0}=0$, and $f_{j}^{n}$ is an approximation of $f\left(t=t^{n}, v=v_{j}\right)$. At last, we define

$$
\langle g\rangle=\sum_{j=1}^{j_{\max }} g_{j} \Delta v
$$

that is a discrete version of $\int_{\mathbb{R}} g(v) d v$.

\section{The explicit Chang and Cooper scheme}

The explicit Chang and Cooper scheme applied to (1.6) is defined by

$$
\frac{1}{\Delta t}\left(f_{j}^{n+1}-f_{j}^{n}\right)=S\left(f^{n}\right)_{j}
$$

with

$$
\begin{aligned}
S\left(f^{n}\right)_{j}= & \frac{\Omega}{\Delta v}\left[\left(v_{j+1 / 2}-U_{e}\right) \bar{f}_{j+1 / 2}^{n}-\left(v_{j-1 / 2}-U_{e}\right) \bar{f}_{j-1 / 2}^{n}\right] \\
& +\frac{\Omega T_{e}}{m \Delta v^{2}}\left(a_{j} f_{j+1}^{n}-b_{j} f_{j}^{n}+c_{j} f_{j-1}^{n}\right)
\end{aligned}
$$

where $a_{j}=c_{j}=1$ and $b_{j}=2$ except at the boundary of the velocity domain (see below). The discrete distribution $\bar{f}_{j+1 / 2}^{n}$ is an approximation of $f\left(t=t^{n}, v=v_{j+1 / 2}\right)$ defined by [1]:

Definition 2.1. The Chang and Cooper average $\bar{f}_{j+1 / 2}$ of the quantities $f_{j}$ and $f_{j+1}$ is defined by

$$
\bar{f}_{j+1 / 2}=\delta_{j+1 / 2} f_{j}+\left(1-\delta_{j+1 / 2}\right) f_{j+1}
$$

where

$$
\left\{\begin{array}{l}
\delta_{j+1 / 2}=\frac{1}{w_{j+1 / 2}}-\frac{1}{\exp \left(w_{j+1 / 2}\right)-1}, \\
w_{j+1 / 2}=\frac{m \Delta v}{T_{e}}\left(v_{j+1 / 2}-U_{e}\right) .
\end{array}\right.
$$

In order to make the Chang and Cooper scheme conservative, we have to impose the Robin boundary condition

$$
\left(v-U_{e}\right) f+\frac{T_{e}}{m} \partial_{v} f=0
$$

at the boundary of the discrete velocity domain. This is equivalent to defining for the numerical scheme

$$
\left\{\begin{array}{l}
a_{j}=1 \quad \text { if } \quad j \neq j_{\max }, \\
b_{j}=2 \text { if } j \in\left\{2, \ldots, j_{\max }-1\right\} \\
c_{j}=1 \text { if } j \neq 1, \\
b_{1}=b_{j_{\max }}=1 \text { and } a_{j_{\max }}=c_{1}=0
\end{array}\right.
$$


and

$$
\bar{f}_{1 / 2}=\bar{f}_{j_{\max }+1 / 2}:=0 .
$$

We have the following classical conservation property:

Property 2.2. The discrete operator $S(f)$ defined by (2.2), and (2.5), (2.6) verifies $\langle S(f)\rangle=0$, which implies that the explicit scheme (2.1), (2.2) verifies

$$
N^{n+1}=N^{n},
$$

where $N:=\langle f\rangle$ is the macroscopic density.

Of course, this property is not a consequence of the Chang and Cooper average but is a direct consequence of the conservative formulation $(2.2)$ of $S(f)$.

Let us now introduce

$$
\mathcal{M}_{f}=\frac{N}{\sqrt{2 \pi T_{e} / m}} \exp \left[-\frac{m\left(v-U_{e}\right)^{2}}{2 T_{e}}\right]
$$

and

$$
\widehat{\mathcal{M}}_{f, j+1 / 2}=\frac{\mathcal{M}_{f, j} \mathcal{M}_{f, j+1}}{\widehat{\mathcal{M}}_{f, j+1 / 2}},
$$

where $\widetilde{\mathcal{M}}_{f, j+1 / 2}$ is the entropic average of $\mathcal{M}_{f, j}$ and $\mathcal{M}_{f, j+1}$, that is to say,

Definition 2.3. The entropic average $\widetilde{f}_{j+1 / 2}$ of the positive quantities $f_{j}$ and $f_{j+1}$ is defined by

$$
\tilde{f}_{j+1 / 2}=\left\{\begin{array}{cl}
\frac{f_{j+1}-f_{j}}{\log f_{j+1}-\log f_{j}} & \text { if } f_{j} \neq f_{j+1}, \\
f_{j} & \text { otherwise. }
\end{array}\right.
$$

By continuity, we extend this definition by setting $\widetilde{f}_{j+1 / 2}=0$ if $f_{j}=0$ or $f_{j+1}=0$.

Thus, $\widetilde{\mathcal{M}}_{f, j+1 / 2}$ in (2.7) is given by

$$
\widetilde{\mathcal{M}}_{f, j+1 / 2}=\frac{\mathcal{M}_{f, j+1}-\mathcal{M}_{f, j}}{\log \mathcal{M}_{f, j+1}-\log \mathcal{M}_{f, j}} .
$$

The entropic average was introduced to discretize the non-linear ion-electron collision operator $[6,8]$ and the non-linear isotropic ion-ion collision operator [7]. Due to Property 2.2, we have $\mathcal{M}_{f^{n}}=\mathcal{M}_{f^{0}}$ and, thus, $\widehat{\mathcal{M}}_{f^{n}, j+1 / 2}=\widehat{\mathcal{M}}_{f^{0}, j+1 / 2}$ for all $n \in \mathbb{N}$. We have the following result:

Lemma 2.4. When $\bar{f}_{j+1 / 2}^{n}$ is the Chang and Cooper average of $f_{j}^{n}$ and $f_{j+1}^{n}$, the discrete operator $S\left(f^{n}\right)_{j}$ defined by (2.2) can be written as

$$
\begin{aligned}
S\left(f^{n}\right)_{j}=\frac{\Omega T_{e}}{m \Delta v^{2}} & \left\{\widehat{\mathcal{M}}_{f^{0}, j+1 / 2}\left[\left(\frac{f^{n}}{\mathcal{M}_{f^{0}}}\right)_{j+1}-\left(\frac{f^{n}}{\mathcal{M}_{f^{0}}}\right)_{j}\right]\right. \\
& \left.-\widehat{\mathcal{M}}_{f^{0}, j-1 / 2}\left[\left(\frac{f^{n}}{\mathcal{M}_{f^{0}}}\right)_{j}-\left(\frac{f^{n}}{\mathcal{M}_{f^{0}}}\right)_{j-1}\right]\right\}
\end{aligned}
$$


where boundary conditions (2.5), (2.6) are replaced with the boundary conditions

$$
f_{0}^{n}:=f_{1}^{n} \cdot \frac{\mathcal{M}_{f^{0}, 0}}{\mathcal{M}_{f^{0}, 1}} \quad \text { and } \quad f_{j_{\max }+1}^{n}:=f_{j_{\max }}^{n} \cdot \frac{\mathcal{M}_{f^{0}, j_{\max }+1}}{\mathcal{M}_{f^{0}, j_{\max }}}
$$

In other words, the Chang and Cooper average (2.3) makes equivalent at the discrete level the convection-diffusion formulation (1.6) and the non-logarithmic Landau formulation (1.8). Moreover, let us remark that boundary conditions (2.10) are equivalent at the discrete level to the boundary condition

$$
\partial_{v}\left(f / \mathcal{M}_{f^{0}}\right)=0 .
$$

Of course, (2.11) is equivalent at the continuous level to Robin boundary condition (2.4). Let us note that (2.9) shows that when $f^{n}$ is equal to the Maxwellian equilibrium $\mathcal{M}_{f^{0}}$, we have $S\left(f^{n}\right)=0$ which implies that $f^{n+1}=f^{n}$ : the Chang and Cooper scheme was initialy built to verify this property that may be easily deduced from the convection-diffusion formulation (2.2). At last, we immediatly deduce from Lemma 2.4:

Corollary 2.5. When $\bar{f}_{j+1 / 2}^{n}$ is the Chang and Cooper average of $f_{j}^{n}$ and $f_{j+1}^{n}$ and when $f_{j}^{n}=0$, the discrete operator $S\left(f^{n}\right)_{j}$ defined by (2.2) can be written as

$$
S\left(f^{n}\right)_{j}=\frac{\Omega T_{e}}{m \Delta v^{2}}\left(\frac{\widehat{\mathcal{M}}_{f^{0}, j+1 / 2}}{\mathcal{M}_{f^{0}, j+1}} f_{j+1}^{n}+\frac{\widehat{\mathcal{M}}_{f^{0}, j-1 / 2}}{\mathcal{M}_{f^{0}, j-1}} f_{j-1}^{n}\right) .
$$

Thus, for any $\Delta t>0, f_{j}^{n+1}>0$ when, for example, $f_{j+1}^{n}>0$ and $f_{j-1}^{n} \geq 0$.

This result shows that Dirac type initial conditions can be treated with the Chang and Cooper scheme. This property is a direct consequence of the Chang and Cooper average.

The proof of Lemma 2.4 uses the following property:

Property 2.6. When $\bar{f}_{j+1 / 2}^{n}$ is the Chang and Cooper average of $f_{j}^{n}$ and $f_{j+1}^{n}$, we can define $\bar{f}_{j+1 / 2}^{n}$ with

$$
\bar{f}_{j+1 / 2}^{n}=\frac{f_{j+1}^{n}-f_{j}^{n}}{\log \mathcal{M}_{f^{0}, j+1}-\log \mathcal{M}_{f^{0}, j}}+\left(\frac{f_{j}^{n}}{\mathcal{M}_{f^{0}, j}}-\frac{f_{j+1}^{n}}{\mathcal{M}_{f^{0}, j+1}}\right) \frac{\mathcal{M}_{f^{0}, j+1} \mathcal{M}_{f^{0}, j}}{\mathcal{M}_{f^{0}, j+1}-\mathcal{M}_{f^{0}, j}} .
$$

Proof of Lemma 2.4. By using (2.13), we obtain that when $j \in\left\{2, \ldots, j_{\max }-1\right\}$

$$
\begin{aligned}
S\left(f^{n}\right)_{j}= & \frac{\Omega}{\Delta v}\left(\frac{f_{j}^{n}}{\mathcal{M}_{f^{0}, j}}-\frac{f_{j+1}^{n}}{\mathcal{M}_{f^{0}, j+1}}\right) \frac{\mathcal{M}_{f^{0}, j+1} \mathcal{M}_{f^{0}, j}}{\mathcal{M}_{f^{0}, j+1}-\mathcal{M}_{f^{0}, j}}\left(v_{j+1 / 2}-U_{e}\right) \\
& -\frac{\Omega}{\Delta v}\left(\frac{f_{j-1}^{n}}{\mathcal{M}_{f^{0}, j-1}}-\frac{f_{j}^{n}}{\mathcal{M}_{f^{0}, j}}\right) \frac{\mathcal{M}_{f^{0}, j} \mathcal{M}_{f^{0}, j-1}}{\mathcal{M}_{f^{0}, j}-\mathcal{M}_{f^{0}, j-1}}\left(v_{j-1 / 2}-U_{e}\right) \\
& +\frac{\Omega}{\Delta v}\left[\frac{f_{j+1}^{n}-f_{j}^{n}}{\log \mathcal{M}_{f^{0}, j+1}-\log \mathcal{M}_{f^{0}, j}}\left(v_{j+1 / 2}-U_{e}\right)\right. \\
& \left.-\frac{f_{j}^{n}-f_{j-1}^{n}}{\log \mathcal{M}_{f^{0}, j}-\log \mathcal{M}_{f^{0}, j-1}}\left(v_{j-1 / 2}-U_{e}\right)\right] \\
& +\frac{\Omega T_{e}}{m \Delta v^{2}}\left(f_{j+1}^{n}-2 f_{j}^{n}+f_{j-1}^{n}\right) .
\end{aligned}
$$


But, since $v_{j+1}=v_{j}+\Delta v$ and $v_{j+1 / 2}=\left(v_{j}+v_{j+1}\right) / 2$, we also have

$$
w_{j+1 / 2}:=\frac{m \Delta v}{T_{e}}\left(v_{j+1 / 2}-U_{e}\right)=-\left[\left(\log \mathcal{M}_{f^{0}}\right)_{j+1}-\left(\log \mathcal{M}_{f^{0}}\right)_{j}\right] .
$$

Thus, we can write that

$$
\frac{\mathcal{M}_{f^{0}, j+1} \mathcal{M}_{f^{0}, j}}{\mathcal{M}_{f^{0}, j+1}-\mathcal{M}_{f^{0}, j}}\left(v_{j+1 / 2}-U_{e}\right)=-\frac{T_{e}}{m \Delta v} \widehat{\mathcal{M}}_{f^{0}, j+1 / 2}
$$

and that

$$
\frac{\left(v_{j+1 / 2}-U_{e}\right)}{\log \mathcal{M}_{f^{0}, j+1}-\log \mathcal{M}_{f^{0}, j}}=-\frac{T_{e}}{m \Delta v} .
$$

Then, we have

$$
\begin{aligned}
S\left(f^{n}\right)_{j}= & \frac{\Omega T_{e}}{m \Delta v^{2}}\left[\left(\frac{f_{j+1}^{n}}{\mathcal{M}_{f^{0}, j+1}}-\frac{f_{j}^{n}}{\mathcal{M}_{f^{0}, j}}\right) \widehat{\mathcal{M}}_{f^{0}, j+1 / 2}\right. \\
& \left.-\left(\frac{f_{j}^{n}}{\mathcal{M}_{f^{0}, j}}-\frac{f_{j-1}^{n}}{\mathcal{M}_{f^{0}, j-1}}\right) \widehat{\mathcal{M}}_{f^{0}, j-1 / 2}\right] \\
& -\frac{\Omega T_{e}}{m \Delta v^{2}}\left(f_{j+1}^{n}-2 f_{j}^{n}+f_{j-1}^{n}\right)+\frac{\Omega T_{e}}{m \Delta v^{2}}\left(f_{j+1}^{n}-2 f_{j}^{n}+f_{j-1}^{n}\right)
\end{aligned}
$$

that is to say

$$
\begin{aligned}
S\left(f^{n}\right)_{j}=\frac{\Omega T_{e}}{m \Delta v^{2}} & \left\{\widehat{\mathcal{M}}_{f^{0}, j+1 / 2}\left[\left(\frac{f^{n}}{\mathcal{M}_{f^{0}}}\right)_{j+1}-\left(\frac{f^{n}}{\mathcal{M}_{f^{0}}}\right)_{j}\right]\right. \\
& \left.-\widehat{\mathcal{M}}_{f^{0}, j-1 / 2}\left[\left(\frac{f^{n}}{\mathcal{M}_{f^{0}}}\right)_{j}-\left(\frac{f^{n}}{\mathcal{M}_{f^{0}}}\right)_{j-1}\right]\right\} .
\end{aligned}
$$

If $j \in\left\{1, j_{\max }\right\}$, we verify that the equality (2.9) remains true when

$$
f_{0}^{n}:=f_{1}^{n} \cdot \frac{\mathcal{M}_{f^{0}, 0}}{\mathcal{M}_{f^{0}, 1}} \quad \text { and } \quad f_{j_{\max }+1}^{n}:=f_{j_{\max }}^{n} \cdot \frac{\mathcal{M}_{f^{0}, j_{\max }+1}}{\mathcal{M}_{f^{0}, j_{\max }}} .
$$

Proof of Property 2.6. By using (2.14), we obtain that

$$
\delta_{j+1 / 2}=-\frac{1}{\log \mathcal{M}_{f^{0}, j+1}-\log \mathcal{M}_{f^{0}, j}}+\frac{\mathcal{M}_{f^{0}, j+1}}{\mathcal{M}_{f^{0}, j+1}-\mathcal{M}_{f^{0}, j}} .
$$

Then

$$
\bar{f}_{j+1 / 2}^{n}=\frac{f_{j+1}^{n}-f_{j}^{n}}{\log \mathcal{M}_{f^{0}, j+1}-\log \mathcal{M}_{f^{0}, j}}+\frac{f_{j}^{n}-f_{j+1}^{n}}{\mathcal{M}_{f^{0}, j+1}-\mathcal{M}_{f^{0}, j}} \mathcal{M}_{f^{0}, j+1}+f_{j+1}^{n}
$$

which shows that

$$
\bar{f}_{j+1 / 2}^{n}=\frac{f_{j+1}^{n}-f_{j}^{n}}{\log \mathcal{M}_{f^{0}, j+1}-\log \mathcal{M}_{f^{0}, j}}+\left(\frac{f_{j}^{n}}{\mathcal{M}_{f^{0}, j}}-\frac{f_{j+1}^{n}}{\mathcal{M}_{f^{0}, j+1}}\right) \frac{\mathcal{M}_{f^{0}, j+1} \mathcal{M}_{f^{0}, j}}{\mathcal{M}_{f^{0}, j+1}-\mathcal{M}_{f^{0}, j}} .
$$




\section{Positivity of the scheme}

Let us define

$$
\left\{\begin{array}{l}
h_{\max }^{n}=\max _{j}\left(\frac{f^{n}}{\mathcal{M}_{f^{0}}}\right)_{j}, \\
h_{\min }^{n}=\min _{j}\left(\frac{f^{n}}{\mathcal{M}_{f^{0}}}\right)_{j} .
\end{array}\right.
$$

We have the following result:

Proposition 3.1. For all initial conditions $\left\{f_{j}^{0}\right\}_{j}$, when $\bar{f}_{j+1 / 2}^{n}$ is the Chang and Cooper average of $f_{j}^{n}$ and $f_{j+1}^{n}$, under the CFL criterion

$$
\Delta t \leq \frac{m \Delta v^{2}}{\Omega T_{e}} \min _{j}\left(\frac{\mathcal{M}_{f^{0}, j}}{\widehat{\mathcal{M}}_{f^{0}, j+1 / 2}+\widehat{\mathcal{M}}_{f^{0}, j-1 / 2}}\right),
$$

then the explicit scheme (2.1), (2.2) verifies the maximum principle

$$
h_{\min }^{n} \leq h_{\min }^{n+1} \leq h_{\max }^{n+1} \leq h_{\max }^{n} .
$$

Thus, for all non-negative initial condition $\left\{f_{j}^{0}\right\}_{j}$, the scheme (2.1), (2.2) verifies

$$
\inf _{j, n} f_{j}^{n} \geq 0
$$

this inequality being strict when the initial condition $\left\{f_{j}^{0}\right\}_{j}$ is positive.

This result proves that the numerical scheme (2.1), (2.2) preserves the positivity of the distribution $f_{j}^{n}$ under a CFL criterion. But, it also proves that there exists $h_{\min }^{\infty}$ and $h_{\max }^{\infty}$ such that the sequences $\left\{h_{\min }^{n}\right\}_{n}$ and $\left\{h_{\max }^{n}\right\}_{n}$ admit the respective limits $h_{\min }^{\infty}$ and $h_{\max }^{\infty}$ when $n$ goes to $+\infty$. Let us underline that we cannot deduce from this result that $h_{\min }^{\infty}=h_{\max }^{\infty}$, equality which would imply that there would exist a constant $C>0$ such that

$$
\forall j: \lim _{n \rightarrow \infty} f_{j}^{n}=C \cdot \mathcal{M}_{f^{0}, j} .
$$

Here, we can just deduce from (3.2) that $h_{\min }^{\infty} \leq h_{\max }^{\infty}$. In the sequel, we will deduce the equality between $h_{\min }^{\infty}$ and $h_{\max }^{\infty}$ from the fact that the scheme (2.1) (2.2) is entropy satisfying under a CFL criterion that is more restrictive than (3.1).

Proof of Proposition 3.1. By defining $h_{j}^{n}=f_{j}^{n} / \mathcal{M}_{f^{0}, j}$ and by using Lemma 2.4, we can write

$$
f_{j}^{n+1}=f_{j}^{n}+\frac{\Delta t \Omega T_{e}}{m \Delta v^{2}}\left[\widehat{\mathcal{M}}_{f^{0}, j+1 / 2}\left(h_{j+1}^{n}-h_{j}^{n}\right)-\widehat{\mathcal{M}}_{f^{0}, j-1 / 2}\left(h_{j}^{n}-h_{j-1}^{n}\right)\right] .
$$

Then, it is obvious that

$$
h_{j}^{n}-\frac{\Delta t \Omega T_{e}}{m \Delta v^{2}} \cdot \frac{\widehat{\mathcal{M}}_{f^{0}, j+1 / 2}+\widehat{\mathcal{M}}_{f^{0}, j-1 / 2}}{\mathcal{M}_{f^{0}, j}}\left(h_{j}^{n}-h_{\text {min }}^{n}\right) \leq h_{j}^{n+1}
$$

and that

$$
h_{j}^{n+1} \leq h_{j}^{n}+\frac{\Delta t \Omega T_{e}}{m \Delta v^{2}} \cdot \frac{\widehat{\mathcal{M}}_{f^{0}, j+1 / 2}+\widehat{\mathcal{M}}_{f^{0}, j-1 / 2}}{\mathcal{M}_{f^{0}, j}}\left(h_{\max }^{n}-h_{j}^{n}\right) .
$$


Let us now suppose that $\Delta t$ is such that

$$
\Delta t \leq \frac{m \Delta v^{2}}{\Omega T_{e}} \min _{j}\left(\frac{\mathcal{M}_{f^{0}, j}}{\widehat{\mathcal{M}}_{f^{0}, j+1 / 2}+\widehat{\mathcal{M}}_{f^{0}, j-1 / 2}}\right)
$$

Then, we obtain that

$$
\forall j: h_{\min }^{n} \leq h_{j}^{n+1} \leq h_{\max }^{n}
$$

that is to say

$$
h_{\min }^{n} \leq h_{\min }^{n+1} \leq h_{\max }^{n+1} \leq h_{\max }^{n}
$$

\section{Convergence toward a Maxwellian equilibrium}

Let us now define

$$
\Delta t^{n}=\Delta t^{*} \frac{h_{\min }^{n}}{h_{\max }^{n}}
$$

with

$$
\left\{\begin{array}{l}
\Delta t^{*}=\frac{m}{4 \Omega T_{e}} \cdot \frac{\Delta v^{2}}{M^{0}}, \\
M^{0}=\max _{j}\left(\frac{\mathcal{M}_{f^{0}, j \pm 1}}{\mathcal{M}_{f^{0}, j}}\right) .
\end{array}\right.
$$

We also define the discrete entropy

$$
H^{n}=\left\langle f^{n} \log \left(\frac{f^{n}}{\mathcal{M}_{f^{0}}}\right)\right\rangle .
$$

The following proposition shows that the distribution $f^{n}$ converges toward a Maxwellian equilibrium:

Proposition 4.1. For all positive initial condition $\left\{f_{j}^{0}\right\}_{j}$, when $\bar{f}_{j+1 / 2}^{n}$ is the Chang and Cooper average of $f_{j}^{n}$ and $f_{j+1}^{n}$, under the CFL criterion

$$
\Delta t \leq \Delta t^{n}
$$

the explicit scheme (2.1), (2.2) verifies the inequality

$$
H^{n+1} \leq H^{n},
$$

which implies that

$$
\lim _{n \rightarrow+\infty} \Delta t^{n}=\Delta t^{*}
$$

and that

$$
\lim _{n \rightarrow+\infty} f_{j}^{n}=\frac{N^{0}}{\left\langle\mathcal{M}_{f^{0}, j}\right\rangle} \mathcal{M}_{f^{0}, j}
$$

as soon as $\Delta t>0$. 
This result shows that the Chang and Cooper scheme is entropy satisfying and is a convergent scheme in the case of the linear Fokker-Planck operator (1.6) under the CFL criterion (4.2). We wish to underline that the maximum principle (3.2) and Definition (4.1) show that the sequence $\left\{\Delta t^{n}\right\}_{n \geq 0}$ is an increasing sequence bounded by $\Delta t^{*}$. This means that the CFL criterion (4.2) is less and less restrictive when $n$ goes to infinity. Let us note that we suppose in Proposition 4.1 that the initial condition is positive. In fact, we have almost the same result when the initial condition is only non-negative:

Corollary 4.2. For all non-negative initial condition $\left\{f_{j}^{0}\right\}_{j}$ such that $N^{0}>0$, when $\bar{f}_{j+1 / 2}^{n}$ is the Chang and Cooper average of $f_{j}^{n}$ and $f_{j+1}^{n}$, under a CFL criterion, the explicit scheme (2.1), (2.2) verifies (4.4) and (4.5) as soon as $\Delta t>0$. Nevertheless, if there exists $j_{0}$ such that $f_{j_{0}}^{0}=0$, we can just say that there exists $n_{0} \in \mathbb{N}^{*}$ such that, under the CFL criterion (4.2), (4.3) is satisfied when $n \geq n_{0}$.

This corollary is a consequence of Corollary 2.5. Let us note that Corollary 4.2 does not mean that when $n<n_{0}$, the scheme (2.1), (2.2) cannot be entropy satisfying under a CFL criterion. In fact, Corollary 4.2 means that the approach used in the proof of Proposition 4.1 to prove that the scheme is entropy satisfying under the CFL criterion (4.2) may not be valid when $n<n_{0}$ (we easily understand this point by noting that $\Delta t^{n}=0$ as soon as there exists $j_{0}$ such that $\left.f_{j_{0}}^{n}=0\right)$. Nevertheless, by using the fact that $\lim _{x \rightarrow 0^{+}} x \log x=0$, it should be possible to prove that the scheme is also entropy satisfying under a CFL criterion when $n<n_{0}$.

To prove Proposition 4.1, we use the following lemma.

LEMMA 4.3 .

$$
\forall j: \frac{\mathcal{M}_{f^{0}, j}}{\widehat{\mathcal{M}}_{f^{0}, j+1 / 2}+\widehat{\mathcal{M}}_{f^{0}, j-1 / 2}} \geq \frac{1}{2 M^{0}}
$$

Proof of Proposition 4.1. Let us suppose that $\Delta t>0$ satisfies the CFL criterion (4.2). Thus, by using Lemma 4.3, we obtain that $\Delta t$ also satisfies the CFL criterion (3.1) which implies that $f_{j}^{n+1}>0$ by using Proposition 3.1. As a consequence, we can evaluate the entropy $H^{n+1}$. By using the fact that $f_{j}^{n+1}=f_{j}^{n}+\Delta t S\left(f^{n}\right)_{j}$, we obtain that

$$
\begin{aligned}
H^{n+1}= & \sum_{j}\left[f_{j}^{n}+\Delta t S\left(f^{n}\right)_{j}\right] \log \left(\frac{f_{j}^{n}+\Delta t S\left(f^{n}\right)_{j}}{\mathcal{M}_{f^{0}, j}}\right) \Delta v \\
= & H^{n}+\Delta t \sum_{j} S\left(f^{n}\right)_{j} \log \left(\frac{f^{n}}{\mathcal{M}_{f^{0}}}\right)_{j} \Delta v \\
& +\left[f_{j}^{n}+\Delta t S\left(f^{n}\right)_{j}\right] \log \left(1+\frac{\Delta t S\left(f^{n}\right)_{j}}{f_{j}^{n}}\right) \Delta v .
\end{aligned}
$$

Since $f_{j}^{n+1}>0$, we also have $\frac{\Delta t S\left(f^{n}\right)_{j}}{f_{j}^{n}}>-1$. Thus, by using the inequality

$$
\forall x>-1: \log (1+x)<x
$$


and by using the fact that $\sum_{j} S\left(f^{n}\right)_{j} \Delta v=0$ (see Property 2.2), we obtain that

$$
H^{n+1} \leq H^{n}+\Delta t \sum_{j}\left[S\left(f^{n}\right)_{j} \log \left(\frac{f^{n}}{\mathcal{M}_{f^{0}}}\right)_{j}+\Delta t \frac{S\left(f^{n}\right)_{j}^{2}}{f_{j}^{n}}\right] \Delta v .
$$

By applying the Schwarz inequality to (2.9), we obtain that

$$
\begin{aligned}
S\left(f^{n}\right)_{j}^{2} \leq \frac{\Omega T_{e}}{m \Delta v^{2}}\left(\widehat{\mathcal{M}}_{f^{0}, j+1 / 2}+\widehat{\mathcal{M}}_{f^{0}, j-1 / 2}\right) \frac{\Omega T_{e}}{m \Delta v^{2}} & {\left[\widehat{\mathcal{M}}_{f^{0}, j+1 / 2}\left(h_{j+1}^{n}-h_{j}^{n}\right)^{2}\right.} \\
& \left.+\widehat{\mathcal{M}}_{f^{0}, j-1 / 2}\left(h_{j-1}^{n}-h_{j}^{n}\right)^{2}\right],
\end{aligned}
$$

where we have defined $h_{j}^{n}=f_{j}^{n} / \mathcal{M}_{f^{0}, j}$. And, by using the fact that $\widehat{\mathcal{M}}_{f^{0}, j \pm 1 / 2}>0$ and by using again Lemma 4.3 , we obtain

$$
\sum_{j} \frac{S\left(f^{n}\right)_{j}^{2}}{f_{j}^{n}} \leq \frac{\Omega T_{e}}{m \Delta v^{2}} \cdot \frac{2 M^{0}}{h_{\min }^{n}} \cdot \frac{2 \Omega T_{e}}{m \Delta v^{2}} \sum_{j} \widehat{\mathcal{M}}_{f^{0}, j+1 / 2}\left(h_{j+1}^{n}-h_{j}^{n}\right)^{2} .
$$

By using the fact that $x \mapsto \log x$ is an increasing function, we can write that

$$
\forall x \geq 0, \forall y \geq 0: \frac{x-y}{\log x-\log y} \leq \max (x, y),
$$

which implies that

$$
\sum_{j} \frac{S\left(f^{n}\right)_{j}^{2}}{f_{j}^{n}} \leq \frac{\Omega T_{e}}{m \Delta v^{2}} \cdot \frac{2 M^{0}}{h_{\min }^{n}} \cdot \frac{2 \Omega T_{e}}{m \Delta v^{2}} \sum_{j} \widehat{\mathcal{M}}_{f^{0}, j+1 / 2}\left(h_{j+1}^{n}-h_{j}^{n}\right)\left(\log h_{j+1}^{n}-\log h_{j}^{n}\right) h_{\max }^{n} .
$$

Moreover, by using (2.9), we have

$$
\begin{aligned}
& \sum_{j} S\left(f^{n}\right)_{j} \cdot \log \left(f^{n} / \mathcal{M}_{f^{0}}\right)_{j} \\
= & \frac{\Omega T_{e}}{m \Delta v^{2}} \sum_{j} \widehat{\mathcal{M}}_{f^{0}, j+1 / 2}\left[\left(f^{n} / \mathcal{M}_{f^{0}}\right)_{j+1}-\left(f^{n} / \mathcal{M}_{f^{0}}\right)_{j}\right] \log \left(f^{n} / \mathcal{M}_{f^{0}}\right)_{j} \\
& -\frac{\Omega T_{e}}{m \Delta v^{2}} \sum_{j} \widehat{\mathcal{M}}_{f^{0}, j-1 / 2}\left[\left(f^{n} / \mathcal{M}_{f^{0}}\right)_{j}-\left(f^{n} / \mathcal{M}_{f^{0}}\right)_{j-1}\right] \log \left(f^{n} / \mathcal{M}_{f^{0}}\right)_{j},
\end{aligned}
$$

that is to say

$$
\sum_{j} S\left(f^{n}\right)_{j} \log \left(f^{n} / \mathcal{M}_{f^{0}}\right)_{j}=-\frac{\Omega T_{e}}{m \Delta v^{2}} \sum_{j} \widehat{\mathcal{M}}_{f^{0}, j+1 / 2}\left(h_{j+1}^{n}-h_{j}^{n}\right)\left(\log h_{j+1}^{n}-\log h_{j}^{n}\right) \leq 0
$$

by again using the fact that $x \mapsto \log x$ is an increasing function. Thus, we can write that

$$
\sum_{j} \frac{S\left(f^{n}\right)_{j}^{2}}{f_{j}^{n}} \leq-\frac{4 \Omega T_{e}}{m \Delta v^{2}} \cdot \frac{h_{\max }^{n}}{h_{\min }^{n}} M^{0} \sum_{j} S\left(f^{n}\right)_{j} \log \left(\frac{f^{n}}{\mathcal{M}_{f^{0}}}\right)_{j} .
$$

Finally, we obtain

$$
H^{n+1} \leq H^{n}+\Delta t\left(1-\Delta t \frac{4 \Omega T_{e}}{m \Delta v^{2}} \cdot \frac{h_{\max }^{n}}{h_{\min }^{n}} M^{0}\right) \sum_{j} S\left(f^{n}\right)_{j} \log \left(\frac{f^{n}}{\mathcal{M}_{f^{0}}}\right)_{j} \Delta v .
$$


Then, when $\Delta t<\Delta t^{n}$, by using inequality (4.8) we obtain the inequality

$$
H^{n+1} \leq H^{n}+\Delta t\left(1-\frac{\Delta t}{\Delta t^{n}}\right) \cdot \sum_{j} S\left(f^{n}\right)_{j} \log \left(\frac{f^{n}}{\mathcal{M}_{f^{0}}}\right)_{j} \Delta v \leq H^{n} .
$$

Thus, under CFL criterion (4.2), we obtain that $H^{n}$ is a decreasing sequence. Moreover, (3.2) implies that $f_{j}^{n}$ is bounded. As a consequence, $H^{n}$ is also bounded. This implies that there exists $H^{\infty} \in \mathbb{R}$ such that

$$
\lim _{n \rightarrow+\infty} H^{n}=H^{\infty} .
$$

Moreover, by again using (3.2), we obtain that

$$
\Delta t^{n} \leq \Delta t^{n+1} \leq \Delta t^{*}
$$

that is to say

$$
\inf _{n} \Delta t^{n}>0
$$

which means that we can choose $\Delta t$ at any time $t^{n}$ in such a way $\Delta t \geq \varepsilon$ with $\varepsilon>0$. Thus, when $H^{n+1}=H^{n}=H^{\infty},(4.9),(4.10)$, and (4.11) imply that

$$
\sum_{j} S\left(f^{n}\right)_{j} \log \left(\frac{f^{n}}{\mathcal{M}_{f^{0}}}\right)_{j} \Delta v=0
$$

that is to say

$$
\forall j: h_{j+1}^{n}=h_{j}^{n}
$$

by using (4.8). We deduce from this equality limit (4.4) and that

$$
\forall j: \lim _{n \rightarrow+\infty} f_{j}^{n}=C \cdot \mathcal{M}_{f^{0}, j},
$$

where $C \in \mathbb{R}^{*}$. The mass conservation Property 2.2 allows to write that $C=\frac{N^{0}}{\left\langle\mathcal{M}_{f^{0}, j}\right\rangle}$.

Proof of Lemma 4.3. We have

$$
\frac{\widehat{\mathcal{M}}_{f^{0}, j+1 / 2}+\widehat{\mathcal{M}}_{f^{0}, j-1 / 2}}{\mathcal{M}_{f^{0}, j}}=\frac{\log \left(\mathcal{M}_{f^{0}, j} / \mathcal{M}_{f^{0}, j+1}\right)}{\mathcal{M}_{f^{0}, j} / \mathcal{M}_{f^{0}, j+1}-1}+\frac{\log \left(\mathcal{M}_{f^{0}, j} / \mathcal{M}_{f^{0}, j-1}\right)}{\mathcal{M}_{f^{0}, j} / \mathcal{M}_{f^{0}, j-1}-1} .
$$

Then

$$
\frac{\widehat{\mathcal{M}}_{f^{0}, j+1 / 2}+\widehat{\mathcal{M}}_{f^{0}, j-1 / 2}}{\mathcal{M}_{f^{0}, j}} \leq \frac{1}{\min \left(1, \mathcal{M}_{f^{0}, j} / \mathcal{M}_{f^{0}, j+1}\right)}+\frac{1}{\min \left(1, \mathcal{M}_{f^{0}, j} / \mathcal{M}_{f^{0}, j-1}\right)}
$$

since $\forall x \geq 0: \min (1, x) \leq(x-1) / \log x$. But

$$
\begin{aligned}
\frac{1}{\min \left(1, \mathcal{M}_{f^{0}, j} / \mathcal{M}_{f^{0}, j+1}\right)}+\frac{1}{\min \left(1, \mathcal{M}_{f^{0}, j} / \mathcal{M}_{f^{0}, j-1}\right)} & \leq \frac{2}{\min _{k}\left(\mathcal{M}_{f^{0}, k \pm 1} / \mathcal{M}_{f^{0}, k}\right)} \\
& =2 \max _{k}\left(\mathcal{M}_{f^{0}, k \pm 1} / \mathcal{M}_{f^{0}, k}\right) \\
& =2 M^{0}
\end{aligned}
$$

which gives the result. 


\section{Conclusion}

We have shown that for a particular linear Fokker-Planck operator that is a linear version of an ion-electron collision operator, the explicit Chang and Cooper scheme [1] has good properties. Indeed, when the initial condition is positive, and under a classical CFL criterion, the entropy decreases, the distribution is positive and it converges toward a Maxwellian equilibrium. The proof is based on the fact that, at the discrete level, the convection-diffusion formulation of the studied linear FokkerPlanck operator is equivalent to a non-logarithmic Landau formulation in the case of the Chang and Cooper scheme. For this Fokker-Planck operator, it seems to be difficult to obtain similar results in the non-linear case whereas it is possible to obtain such results by replacing the Chang and Cooper average by the entropic average $[6,7,8]$. Nevertheless, the Chang and Cooper scheme is interesting also in the nonlinear case because it is easy to propose an implicit version of this scheme. At last, it should be interesting to try to apply the proposed approach to other linear FokkerPlanck operators as those coming from the Wilkins model of neutron thermalization [9] or from a linearization of the Compton (and inverse Compton) operator [10] (see also the introduction in [2] for these models).

\section{REFERENCES}

[1] J.S. Chang and G. Cooper, A practical difference scheme for Fokker-Planck equations, J. Comput. Phys., 6, 1-16, 1970.

[2] E.W. Larsen, C.D. Levermore, G.C. Pomraning and J.G. Sanderson, Discretization methods for one-dimensional Fokker-Planck operators, J. Comput. Phys., 61, 359-390, 1985.

[3] D. Deck and S. Samba, Le Code Procions, Internal report CEA-DAM, no , 2561, 1994.

[4] E.M. Epperlein, Implicit and conservative difference scheme for the Fokker-Planck equation, J. Comput. Phys., 112, 291-297, 1994.

[5] V.A. Mousseau and D.A. Knoll, Fully implicit kinetic solution of collisional plasmas, J. Comput. Phys., 136, 308-323, 1997.

[6] S. Dellacherie, Sur un schéma numérique semi-discret appliqué un opérateur de Fokker-Planck isotrope, C.R. Acad. Sci. Paris, série I, 328, 1219-1224, 1999.

[7] C. Buet, S. Dellacherie and R. Sentis, Numerical solution of an ionic Fokker-Planck equation with electronic temperature, SIAM J. Numer. Anal., 39(4), 1219-1253, 2001.

[8] S. Dellacherie, Numerical resolution of an ion-electron collision operator in axisymmetrical geometry, Transp. Theory and Stat. Phys., 31, 397-429, 2002.

[9] M.M.R. Williams, The Slowing Down and Thermalization of Neutrons, North-Holland, Amsterdam, 45, 1966.

[10] G.C. Pomraning, The Equations of Radiation Hydrodynamics, Pergamon, Oxford, 191, 1973. 